\title{
A single mitochondrial DNA deletion accurately detects significant prostate cancer in men in the PSA 'grey zone'
}

\author{
Jennifer Creed ${ }^{1,2} \cdot$ Laurence Klotz $^{3} \cdot$ Andrew Harbottle $^{1,2} \cdot$ Andrea Maggrah $^{1,2} \cdot$ Brian Reguly $^{2} \cdot$ Anne George $^{5}$. \\ Vincent Gnanapragasm ${ }^{4,5}$
}

Received: 16 September 2017 / Accepted: 7 December 2017 / Published online: 16 December 2017

(c) The Author(s) 2017. This article is an open access publication

\begin{abstract}
Purpose To determine the clinical performance of a blood-based test for clinically significant (CS) prostate cancer (PCa) (grade group $\geq 2$ ) intended for use in men with prostate serum antigen levels in the 'grey zone' (PSA $<10 \mathrm{ng} / \mathrm{ml}$ ). The test quantifies a previously described $3.4 \mathrm{~kb}$ mitochondrial DNA (mtDNA) deletion.

Methods In a first prospective study of an MRI-guided re-biopsy population $(n=126)$, the $3.4 \mathrm{~kb}$ deletion and $18 \mathrm{~S}$ rRNA gene were amplified from plasma. A diagnostic threshold was selected from the coordinates of the receiver operating characteristic curve and tested in a second population of men who were $(n=92)$ biopsy naïve when the mtDNA deletion was assayed and for whom those diagnosed with cancer on initial biopsy were treated with radical prostatectomy.

Results The $3.4 \mathrm{~kb}$ deletion was a good predictor of CS PCa in the image-guided re-biopsy population [AUC 0.84, (95\% CI 0.73-0.95)] and the selected threshold corresponded to a sensitivity of $87 \%$ [95\% CI, 70-96\%], specificity of $68 \%$ [95\% CI, 47-85\%] and negative predictive value (NPV) of 97\%. Applying this threshold to the second population showed this deletion to be a strong predictor of CS cancer [AUC 0.98, (95\% CI 0.94-1.02)], independent of PSA or age [sensitivity $100 \%$ (95\% CI, 93-100\%), specificity $90 \%$ (95\% CI 73-98\%) and NPV 100\%].

Conclusion The $3.4 \mathrm{~kb}$ deletion in plasma is an accurate predictor of CS cancer for men in the PSA 'grey zone'. Used in advance of biopsy for improved patient selection, this deletion may reduce the number of biopsies needed to diagnose CS prostate cancers.
\end{abstract}

Keywords Mitochondrial DNA · Prostate cancer - Multi-parametric magnetic resonance imaging · Plasma · Biomarker · DNA deletion $\cdot$ Real-time PCR $\cdot$ Diagnostic accuracy

\section{Introduction}

Electronic supplementary material The online version of this article (https://doi.org/10.1007/s00345-017-2152-z) contains supplementary material, which is available to authorized users.

Jennifer Creed

j.creed@mdnalifesciences.com

MDNA Life Sciences UK Ltd, Newcastle upon Tyne, UK

2 MDNA Life Sciences Inc, 2054 Vista Parkway, Suite 400, West Palm Beach, FL 33411, USA

3 Division of Urology, Sunnybrook Health Sciences Centre, University of Toronto, Toronto, Canada

4 Academic Urology Group, Department of Surgery and Oncology, University of Cambridge, Cambridge, UK

5 Cambridge Urology Translational Research and Clinical Trials, Cambridge Biomedical Campus, Cambridge, UK
Indication for prostate biopsy due to suspicion of prostate cancer is primarily driven by elevated levels of serum prostate-specific antigen (PSA) [1, 2]. While inexpensive and accessible, effective use of PSA for screening is limited by its low diagnostic accuracy [3]. Further, most PSA measurements are within the PSA 'grey zone', $<10 \mathrm{ng} / \mathrm{ml}$ where accuracy is much lower [4]. Thus, the identification of a prostate cancer biomarker that can be measured in blood, provided at low cost, is independent of PSA, and accurately identifies CS prostate cancers that would benefit from nearterm intervention, is a pressing need.

We and others have previously reported the clinical value of a $3.4 \mathrm{~kb}$ mitochondrial DNA (mtDNA) deletion (Online Resource 1) strongly correlated with the presence of prostate cancer and elevated even in normal-appearing 
prostate cells coexisting with malignant cells-exhibiting the concept of field cancerization [5-8]. Using laser capture micro-dissection, we reported that the quantity of the deletion while significantly elevated in malignant prostate cells, was not elevated in benign prostate conditions such as hyperplasia and inflammation [5]. As a biomarker derived from the mitochondrial genome, this deletion has several inherent biological traits that confer an advantage over its nuclear genome counterparts [9-11]. Briefly, mitochondrial genome deletions are heteroplasmic [9] and cellular energy production can be maintained even with a significant proportion of mutated mitochondrial genomes [9]. Thus, the mutated deleted species can persist in the cell instead of being repaired, increasing the window of time for detection. The subcellular location of mtDNA within the mitochondrial matrix positions it on the front line of cellular stress responses such as reactive oxygen species production, thus mtDNA mutation events can be an early indicator of the disease [10]. Mitochondrial alterations can contribute directly to tumour cell progression and metastasis, meaning, these mutations can be closely entwined with the disease itself [12]. Finally, mtDNA outnumber the nuclear genome in each cell by hundreds to thousands of copies, translating to robust detection in samples with even limited cellularity such as blood plasma [11].

Here we have focused on determining the diagnostic accuracy of the $3.4 \mathrm{~kb} \mathrm{mtDNA}$ deletion in its cell-free circulating DNA form for men in the PSA 'grey zone' (PSA $<10 \mathrm{ng}$ / $\mathrm{ml})$ to detect CS cancer, defined as grade group 2 or greater (Gleason score 7 or greater). In this study, we defined a diagnostic threshold in an image-guided re-biopsy population and tested its performance in a second population of men who were biopsy naïve at the time of blood collection. Men diagnosed with prostate cancer in the second population were diagnosed on first biopsy and treated with radical prostatectomy, thus deletion levels were correlated to final surgical pathology.

\section{Methods}

\section{Study cohorts}

\section{Image-guided re-biopsy population}

Patients and samples were recruited from prostate cancer clinics at the Department of Urology, Cambridge University Hospitals Trust (CUHT), Cambridge UK [Ethics (Ethics 03/018-NRES Committee East of England, UK)]. All men previously had a PSA $<10 \mathrm{ng} / \mathrm{ml}$, at least one prior negative prostate biopsy and required re-biopsy because of ongoing suspicion of cancer. Blood was collected for testing prior to MRI-guided repeat biopsy. All had a mpMRI and proceeded to trans-perineal template biopsy regardless of whether a target lesion was identified or not. Patients underwent mpMRI on a 1.5T MR450 or 3T Discovery MR750-HDx system (GE Healthcare, USA) with a multi-channel surface phased array coil, including standard anatomical and functional diffusion-weighted imaging using multiple b-values, as previously described [13]. T2W and DWI sequences were evaluated and scored using a Likert scale of cancer probability, based on the prostate imaging reporting and data system (PI-RADS) descriptors developed by the European Society of Urogenital Radiology (ESUR) [14]. The contours of mpMRI defined lesions (Likert 3-5) were drawn on the BiopseeTM fusion platform (Medcom, Germany). No lesion or Likert 1-3 lesions were considered mpMRI negative for this study. 24 sectoral biopsies +2 from each target area using image fusion guidance (where applicable) were taken using a previously published standardized template [13]. Men without mpMRI lesions (mpMRI negative) had only 24 sectoral biopsies taken. Biopsy samples were processed through a single pathology laboratory and reported by specialist uropathologists (using ISUP 2005) [15].

\section{First biopsy cohort}

Patients and samples were recruited by the Ontario Tumour Bank (Toronto, Canada) and Bioreclamation IVT (Westbury, New York, USA). All men were biopsy naïve at the time of blood collection. For cancer positive cases the inclusion criteria were (1) men diagnosed with cancer on first biopsy (2) presenting with PSA $<10 \mathrm{ng} / \mathrm{ml}$, (3) plasma collected pre-biopsy and (4) treated with a radical prostatectomy with final surgical histology available. Age at the time of blood collection was recorded as within 5-year intervals, thus for analysis, the median value of the interval was used. Biological materials were provided by the Ontario Tumour Bank. As control (non-cancer) samples were not available from the Ontario Tumour Bank, male control samples were obtained from Bioreclamation IVT. PSA levels were not available for these men, however, at the time of enrolment none had a prior diagnosis of prostate cancer and were not in a urologist's care due to suspicion of prostate cancer as verified during donor screening by Bioreclamation IVT.

\section{Sample handling and processing}

Blood samples were collected in K2EDTA and plasma was separated by centrifugation and frozen until DNA extraction. DNA was isolated from $200 \mu \mathrm{l}$ plasma using the QIAamp 96 QIAcubeHT extraction kit (Qiagen, Crawley, UK). The 18S rRNA gene and $3.4 \mathrm{~kb}$ deletion were quantified using quantitative polymerase chain reaction. Detailed methods and assay performance are provided in Online Resource 2. 
$P C R$ reagent kit-The $\mathrm{PCR}$ mixture, positive controls, and primers for both $18 \mathrm{~S}$ rRNA and the $3.4 \mathrm{~kb}$ deletion for detection in DNA extracted from blood plasma will be available as a Research Use Only (RUO) reagent kit in 2018. This will allow any third party to continue independent investigations using the same high-quality reagents and optimized assay parameters established by MDNA. The availability of the RUO reagent kit is intended to achieve better quality control and limit interlaboratory assay variability.

\section{Statistical analysis}

All samples and targets were amplified in triplicate, and average $\mathrm{Cq}$ values were calculated. The normalised $3.4 \mathrm{~kb}$ deletion value $(\Delta \mathrm{Cq})$ was determined by relative quantification of the $3.4 \mathrm{~kb}$ deletion to the $18 \mathrm{~S}$ rRNA reference gene. Statistical analyses were performed using Graphpad Prism 5.0, (Graphpad software Inc, La Jolla, CA, USA) for receiver operating characteristic (ROC) curves and descriptives. SPSS v.17.0 (IBM Corp., Armonk, NY, USA) was used for all correlations and tests of significance. The clinical characteristics were summarized using count and percentages for the categorical data, and the mean, standard deviation, and range for continuous variables. The means of two groups were compared using the Student's $t$ test and the Mann-Whitney $U$ test for parametric and non-parametric distributions, respectively. The correlation between the two variables was assessed with the Pearson correlation coefficient $(r)$. With respect to the presence of CS prostate cancer, ROC curves were constructed for the $3.4 \mathrm{~kb}$ mtDNA deletion. The area under the curve (AUC) of the ROC and the sensitivity and specificity at selected cut-offs were calculated with $95 \%$ confidence intervals $(95 \% \mathrm{CI})$. A $p$ value $<0.05$ was considered statistically significant for all tests. For men in the CUHT cohort, the final pathological outcome was based on the image-guided re-biopsy, where men with two negative biopsies and a negative MRI were considered negative controls (MRI-/Bx-). For men in the biopsy naive cohort, outcome was based upon grade group determined at prostatectomy. Men in the control group did not have surgical outcomes and were considered negative due to the absence of clinical suspicion of prostate cancer.

\section{Results}

\section{Performance of the $3.4 \mathrm{~kb}$ mtDNA deletion in an MRI-guided re-biopsy population}

\section{Patient characteristics}

The clinical and demographic characteristics of the 126 participants in this cohort are summarized in Online Resource
3. Seventy-six $(60.3 \%)$ had a diagnosis of prostate cancer at the close of the study, with $31(24.6 \%)$ having CS cancer. Of men without a diagnosis of cancer, $25(50 \%)$ were both histologically and MRI negative [MRI $(-) / \mathrm{Bx}(-)]$. The mean ( \pm s.d.) age for all patients, all cancer patients, all CS cancer patients, and all MRI(-)/Bx(-) controls was 63.77 ( \pm 6.55$)$, $64.51( \pm 6.18), 65.10( \pm 5.90), 61.80( \pm 7.57)$, respectively. The mean $( \pm$ s.d.) PSA for all patients, all cancer patients, all CS cancer patients, and all $\mathrm{MRI}(-) / \mathrm{Bx}(-)$ controls was $6.38( \pm 4.09), 6.63( \pm 4.93), 5.78( \pm 3.37), 6.21( \pm 2.30)$, respectively. The difference between groups for age and PSA was not statistically significant.

\section{4 kb mitochondrial DNA deletion quantity for prediction of CS cancers}

The distribution of the $3.4 \mathrm{~kb}$ deletion $(\Delta \mathrm{Cq})$ among MRI-/ $\mathrm{Bx}$ - control samples, MRI +/Bx-, GS6 and CS cancers is shown in Fig. 1a. The mean ( \pm s.d.) $\Delta \mathrm{Cq}$ for control samples was 10.67 ( \pm 2.429$)$, while the mean ( \pm s.d.) $\Delta$ Cq for CS PCa patients was $8.16( \pm 1.96)$. This difference was significant at $p<0.0001$. $3.4 \mathrm{~kb}$ deletion levels in the MRI $+/ \mathrm{Bx}-$ and grade group 1 prostate cancers were both intermediate between the controls and CS cancers with means of 9.61 $( \pm 2.75)$ and $8.94( \pm 1.96)$ respectively. The nuclear DNA $18 \mathrm{~S}$ rRNA gene used for normalisation of the deletion quantity did not vary significantly between groups $(p=0.170)$ with a mean ( \pm s.d.) 25.5 ( \pm 1.627) for controls and mean ( \pm s.d.) 26.01 ( \pm 0.914) for CS cancers. Neither PSA nor age were significantly correlated with $3.4 \mathrm{~kb}$ deletion quantity, $r=-0.052$ and 0.038 , respectively. The $3.4 \mathrm{~kb}$ deletion for detection of CS cancers $(n=31)$ produced an AUC of 0.84 [95\% CI, 0.73-0.95], $p<0.0001$, when compared to levels detected in the control population $(n=25)$ (Fig. 1b). Using the ROC coordinates a threshold of 9.68 was selected to maximize the number of CS cancers detected while maintaining acceptable specificity and corresponded to a sensitivity of $87 \%$ [95\% CI, 70-96\%], and a specificity of $68 \%$ [95\% CI, 47-85\%].

\section{$3.4 \mathrm{~kb}$ mitochondrial DNA deletion for prediction of any cancer}

The selected diagnostic threshold was applied to determine the accuracy with which participants diagnosed with any grade group prostate cancer could be distinguished from the control group. This yielded an AUC 0.77 [95\% CI, 0.67-0.87], $p<0.0001$ with comparable specificity to the CS cancer at $68 \%$ [95\% CI, 46-85\%] but lower sensitivity at 75\% [95\% CI, 64-84\%], meaning more grade group 1 cancers than CS cancers are considered negative at the selected cut-off. 
Fig. 1 a Distribution of normalised $3.4 \mathrm{~kb}$ mitochondrial DNA deletion values for each clinical subgroup in the repeat biopsy cohort. The horizontal line within the box indicates the median, boundaries of the box indicate the 25 th and 75 th percentile, and the whiskers indicate 10th and 90th percentile of the results. The "+" marked in the box indicates the mean. $G r$ Grp grade group. b Receiver operating characteristic curve of the $3.4 \mathrm{~kb}$ deletion in the repeat biopsy cohort. Normalised $3.4 \mathrm{~kb}$ mtDNA deletion in blood plasma from men in the PSA 'grey zone' showing the accuracy of detection of $\mathrm{CS}(\mathrm{Gr}$ Grp $\geq 2$ ) cancers



b

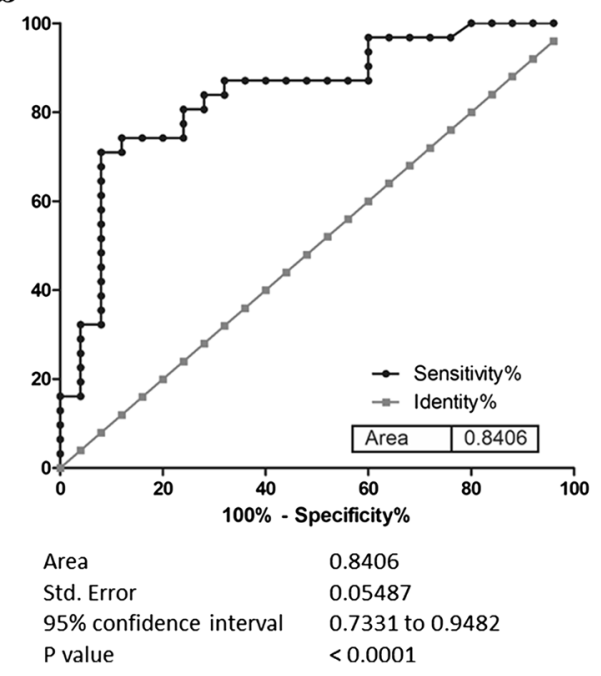

\section{$3.4 \mathrm{~kb}$ mtDNA deletion and MRI}

As all men in this cohort were subject to mpMRI, the accuracy with which the $3.4 \mathrm{~kb}$ deletion could predict an outcome of CS cancer on subsequent biopsy was compared with that of mpMRI using both dichotomous classification of lesion positive or lesion negative, as well as Likert Score. When an indication for biopsy was determined as the detection of a suspicious lesion mpMRI identified $86 \%$ of CS cancers with a specificity of $50 \%$; when restricting indication for biopsy to a Likert Score of 4 or 5 the sensitivity decreased to $74 \%$ with an increase in specificity to $76 \%$. In the subgroup of men with a negative MRI $(n=71)$, defined as no suspicious lesion, the $3.4 \mathrm{~kb}$ deletion appeared predictive of CS cancers [AUC 0.82, (95\% CI, 0.65-0.98), $p<0.0001$ ]. At the defined threshold of 9.68 , the sensitivity was $88 \%$ [95\% CI, 47-100\%] and specificity was 53\% [95\% CI, 36-69\%].
Validation of the $3.4 \mathrm{~kb}$ mitochondrial DNA deletion performance in a first biopsy cohort

\section{Patient characteristics}

The clinical and demographic characteristics for the 92 participants of this cohort are summarized in Online Resource 3. Sixty-three $(68.5 \%)$ had an outcome of prostate cancer and $47(51.1 \%)$ were CS prostate cancer. The mean ( \pm s.d.) age for no cancer controls, those with any cancer, and those with CS cancers were $51.83( \pm 4.49), 62.63( \pm 5.60)$, and 62.53 ( \pm 5.86$)$, respectively. Men in the control group were significantly younger than the men with a diagnosis of cancer $(p<0.0001)$. The mean ( \pm s.d.) PSA for men with any cancer and those with CS cancers was $5.84( \pm 2.22)$ and 6.01 $( \pm 2.14)$, respectively, this difference was not statistically significant $(p=0.69)$. 
Fig. 2 a Distribution of the $3.4 \mathrm{~kb}$ deletion in the first biopsy cohort. The horizontal line within the box indicates the median, boundaries of the box indicate the 25 th and 75 th percentile, and the whiskers indicate 10th and 90th percentile of the results. The "+" marked in the box indicates the mean. $G r$ Grp grade group. b Receiver operating characteristic curve of the $3.4 \mathrm{~kb}$ deletion in the first biopsy cohort. Normalised $3.4 \mathrm{~kb}$ mtDNA deletion in blood plasma from men in the PSA 'grey zone' showing the accuracy of detection of CS ( $\mathrm{Gr}$ Grp $\geq 2$ ) prostate cancers
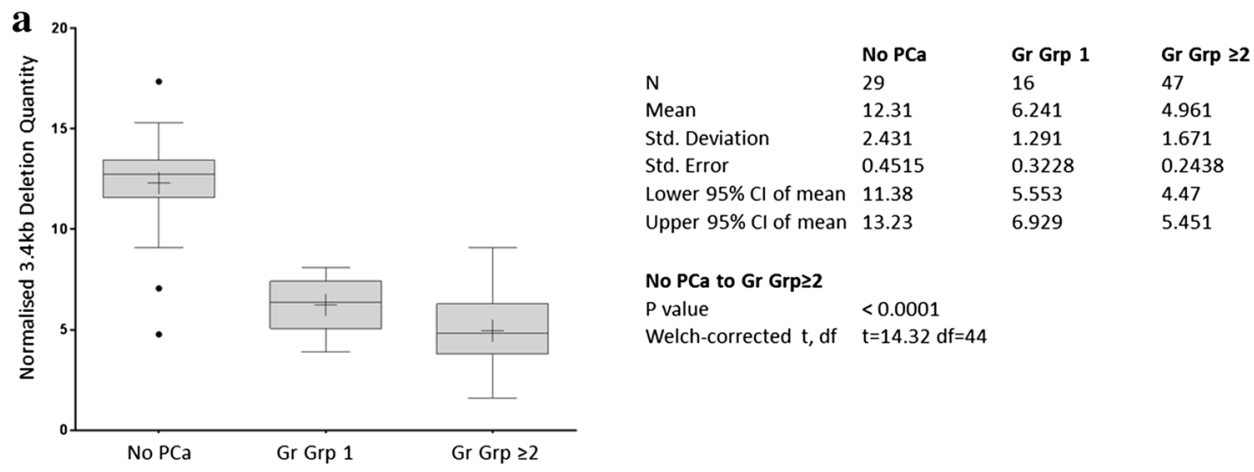

b

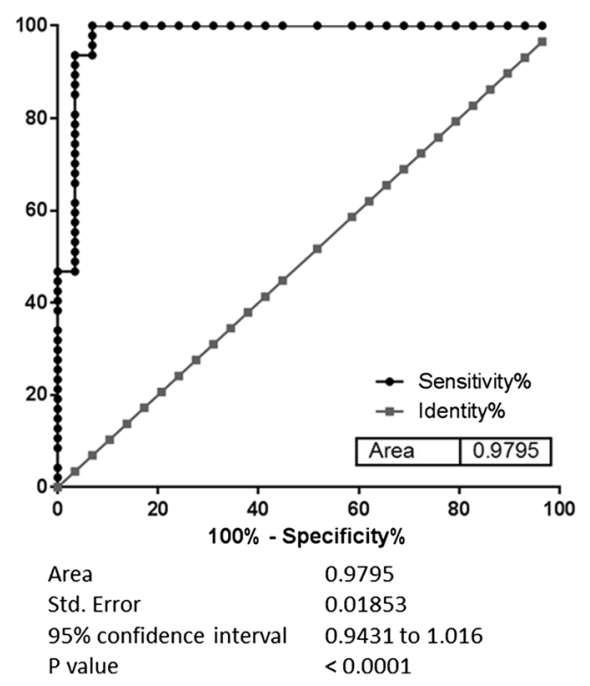

\section{$3.4 \mathrm{~kb}$ mitochondrial DNA deletion quantity for prediction of CS cancers}

The distribution of the $3.4 \mathrm{~kb}$ deletion for each clinical group is shown in Fig. 2a. The mean ( \pm s.d.) $\Delta \mathrm{Ct}$ for control samples was $12.31( \pm 2.43)$, for grade group 1 , 6.24 ( \pm 1.29 ), and for CS prostate cancer patients, 4.96 $( \pm 1.67)$. This difference in deletion quantity between controls and CS cancer was significant at $p<0.0001$. Figure $2 \mathrm{~b}$ shows the diagnostic accuracy of the $3.4 \mathrm{~kb}$ deletion, AUC 0.98 [95\% CI, 0.94-1.02], $p<0.0001$. Applying the threshold of 9.68 for discrimination of CS cancers corresponds to a sensitivity of $100 \%$ [95\% CI, 93-100\%] and a specificity of $90 \%$ [95\% CI, 73-98\%]. This threshold correctly classified all $47 \mathrm{CS}$ cancers and 25 of the 29 controls. All 16 men with grade group 1 cancers in this cohort were classified at this threshold as positive. The NPV and positive predictive value (PPV) were calculated at both the CS cancer incidence rate of the cohort of $62 \%$ as well as downward adjusted to $10 \%$, a figure more consistent with the incidence observed in practice in the intended use population [16]. At both incidence levels, the NPV was $100 \%$ as all CS cancers were correctly classified with the deletion. The PPV was $92.1 \%$ and $44.25 \%$ for the two incidence levels, respectively. No significant correlation was observed between the $3.4 \mathrm{~kb}$ deletion and either PSA $(r=-0.098)$ or age $(r=0.104)$.

\section{Discussion}

This study is the first to report the detection of the $3.4 \mathrm{~kb}$ mitochondrial DNA deletion in plasma and establish and validate a useful diagnostic threshold for the accurate detection of CS prostate cancer for men in the PSA 'grey zone'. Prior reports describe the detection of the $3.4 \mathrm{~kb}$ deletion in prostate tissue, serum and urine and its correlation with prostate cancer [5-7]. However, assay conditions defined for tissue were not optimal for detection in fluids, resulting in an unacceptable failure rate [6]. To optimize detection for use in blood plasma, we reduced the amplicon size for both the $3.4 \mathrm{~kb}$ deletion and the internal control, normalised input DNA using the internal control rather than DNA concentration determined by absorbance at $260 \mathrm{~nm}$, and avoided the use of wild-type mitochondrial DNA as it is widely reported to vary significantly with many other cancers as 
well as aging $[17,18]$ and was expected to negatively impact specificity for prostate cancer. With these optimizations, all samples in this study generated results meeting the defined quality requirements of the assay.

The diagnostic accuracy of the $3.4 \mathrm{~kb}$ deletion in plasma was determined first in an image-guided repeat biopsy cohort [AUC 0.84 (95\% CI, 0.73-0.95), $p<0.0001$ ]. A threshold of $9.68 \Delta \mathrm{Cq}$ was selected from the coordinates of the curve that corresponded to a sensitivity of $87 \%$ [95\% CI, 70-96\%], specificity of $68 \%$ [95\% CI, 47-85\%] and NPV of $97 \%$ for detection of CS cancers. We chose to select the threshold in the repeat biopsy population as the cohort was exceptionally well qualified with both histological and radiological confirmation of disease status. As the gold standard of biopsy is associated with a significant false negative rate $[19,20]$, selection of an optimal diagnostic threshold in a control group defined by histology alone can be expected to include $30-60 \%$ or more undetected cancers $[19,20]$. Thus, determination of the diagnostic threshold in the well-qualified image-guided repeat biopsy cohort reduced this risk [21].

The defined threshold was then tested against a second population of men who were biopsy naïve at the time of plasma collection. In this cohort, men with prostate cancer were diagnosed on first rather than repeat biopsy and were treated surgically with radical prostatectomy. Performance of the $3.4 \mathrm{~kb}$ deletion using the defined threshold was very high, sensitivity of 100\% [95\% CI, 93-100\%], specificity of 90\% [95\% CI, 73-98\%] and NPV of 100\%. Thus, the same diagnostic threshold was effective for the identification of CS cancers in either the repeat biopsy or first biopsy setting though more robust in the latter.

Interestingly, the mean quantity of the $3.4 \mathrm{~kb}$ deletion was significantly higher in men diagnosed on first biopsy compared to the repeat biopsy cohort when stratified by grade group, such that grade group 1 cancers detected on first biopsy had a significantly greater quantity of deletion than did grade group 1 cancers detected on repeat biopsy. The same finding was true for CS cancers. This may suggest that the increase of the $3.4 \mathrm{~kb}$ deletion may be associated not only with decreasing differentiation as seen between well differentiated (grade group 1) and poorly differentiated cancers (grade groups $2+$ ) but perhaps tumour volume as tumours diagnosed on first biopsy are reportedly larger than those diagnosed on repeat biopsy [22, 23].

Compared to other biomarkers of prostate cancer, the $3.4 \mathrm{~kb}$ deletion in a liquid biopsy format has significant advantages. Blood plasma is a common, non-invasive sample matrix amenable to routine testing, and a DNA analyte is more stable than other classes of molecules such as microRNAs [24]. The performance of the biomarker, independent of PSA, supports its use for any man with total PSA in the 'grey zone' who is being considered for biopsy, in contrast to PSA-derived biomarkers which may be contraindicated or require adjustment for men using 5-alpha-reductase inhibitors $[25,26]$. The standard DNA extraction, $\mathrm{qPCR}$ process and result of this single-biomarker assay is simple and costeffective. The assay itself is robust with a negligible failure rate. Finally, the accuracy with which CS prostate cancer within the PSA 'grey zone' is correctly identified or ruled out appears to be unsurpassed by other liquid biopsy biomarkers such as \%fPSA [AUC 0.59 (95\% CI, 0.53-0.65)], phi [AUC 0.64 (95\% CI, 0.59-0.71)], \%p2PSA [AUC 0.64 (95\% CI, 0.58-0.70)] [27], and PCA3 [AUC 0.71 (95\% CI, 0.61-0.81)] [28]. (It should be noted that the lower limit of PSA 'grey zone' varied between these analyses, specifically $2 \mathrm{ng} / \mathrm{ml}$ for \%fPSA, phi, and \%p2PSA, and $4 \mathrm{ng} / \mathrm{ml}$ for PCA3).

In practice, though PSA is a good indicator of prostate cancer at significantly elevated levels $(>10 \mathrm{ng} / \mathrm{ml})$ only a small proportion of all PSA tests performed for screening purposes are above this cut-off [16]. Additionally, there is no lower cut-off for PSA beneath which CS cancers do not occur-up to $15 \%$ of men with PSA less than $4 \mathrm{ng} / \mathrm{ml}$ have CS prostate cancer [29]. This results in a significant number of men in the PSA 'grey zone', with a PSA $<10 \mathrm{ng} / \mathrm{ml}$, and a poorly qualified risk of CS prostate cancer. The diagnostic uncertainty of this group results in a high proportion of unnecessary biopsies and thus a high NPV is of paramount importance for any biomarker intended to better qualify risk in this setting. Ideally, a negative biomarker result must correlate with the absence of CS cancer nearly perfectly. The NPVs reported here (97\% in the repeat biopsy setting, and $100 \%$ in the first biopsy setting) appear to fulfil this requirement well, offering the potential to safely avoid a great many unnecessary biopsies while missing very few CS cancers.

As the $3.4 \mathrm{~kb}$ deletion performed with somewhat better accuracy than mpMRI in this study and was informative for men with a negative mpMRI result, this suggests the deletion may have additional promise complementing the use of mpMRI, however, this requires further study.

The main limitations of this study are the availability of well-qualified control samples for the first biopsy cohort, the use of grade group classifications as a surrogate for clinically significant cancer and the modest sample size.

\section{Conclusion}

The $3.4 \mathrm{~kb}$ mitochondrial DNA deletion measured in blood plasma accurately predicts CS prostate cancer and correlates well to both image-guided biopsy outcome in a repeat biopsy setting as well as prostatectomy outcome in a first biopsy setting for men in the PSA 'grey zone'. As a single biomarker independent of PSA, the test is simple, and the result represents novel information with respect to risk. 
Acknowledgements Ontario Tumour Bank (Ontario, Canada) is supported by the Ontario Institute for Cancer Research through funding provided by the Government of Ontario. VG and AG acknowledge infrastructural support by the Cambridge Biomedical Research campus and Cambridge Cancer Center.

Author contributions JC: Protocol development, Data analysis, Manuscript writing. LK: Manuscript editing. AH: Protocol development, Data analysis, Manuscript writing/editing. AM: Protocol development, Data collection and management, Manuscript writing/editing. BR: Data analysis, Manuscript editing. AG: Sample and data collection. VG: Protocol development, sample collection and clinical data annotation, Manuscript writing/editing.

\section{Compliance with ethical standards}

Conflict of interest JC, AH, AM and BR are employed by or consult for MDNA Life Sciences Inc and are inventors on patents relating to the $3.4 \mathrm{~kb}$ deletion and prostate cancer. VG, AG and LK declare that they have no conflicts of interest.

Funding This study was funded by MDNA Life Sciences Inc.

Ethical approval All procedures performed in studies involving human participants were in accordance with the ethical standards of the institutional and/or national research committee and with the $1964 \mathrm{Hel}-$ sinki declaration and its later amendments or comparable ethical standards. Informed consent was obtained from all individual participants included in the study. Patient specimens were stored and maintained under the Human Tissue Authority (UK) License number\# 12631 for research of human samples.

Open Access This article is distributed under the terms of the Creative Commons Attribution 4.0 International License (http://creativecommons.org/licenses/by/4.0/), which permits unrestricted use, distribution, and reproduction in any medium, provided you give appropriate credit to the original author(s) and the source, provide a link to the Creative Commons license, and indicate if changes were made.

\section{References}

1. Scherr DS, Eastham J, Ohori M, Scardino PT (2002) Prostate biopsy techniques and indications: when, where, and how? Semin Urol Oncol 20(1):18-31

2. Matlaga BR, Eskew LA, McCullough DL (2003) Prostate biopsy: indications and technique. J Urol 169(1):12-19. https://doi. org/10.1097/01.ju.0000041828.84343.53

3. Harvey P, Basuita A, Endersby D, Curtis B, Iacovidou A, Walker M (2009) A systematic review of the diagnostic accuracy of prostate specific antigen. BMC Urol 9:14. https://doi. org/10.1186/1471-2490-9-14

4. Welch HG, Schwartz LM, Woloshin S (2005) Prostate-specific antigen levels in the United States: implications of various definitions for abnormal. J Natl Cancer Inst 97(15):1132-1137. https:// doi.org/10.1093/jnci/dji205

5. Maki J, Robinson K, Reguly B, Alexander J, Wittock R, Aguirre A, Diamandis EP, Escott N, Skehan A, Prowse O, Thayer RE, Froberg MK, Wilson MJ, Maragh S, Jakupciak JP, Wagner PD, Srivastava S, Dakubo GD, Parr RL (2008) Mitochondrial genome deletion aids in the identification of false- and true-negative prostate needle core biopsy specimens. Am J Clin Pathol 129(1):57-66. https://doi.org/10.1309/ujjth4hfepwaq78q

6. Maragh S, Veltri RW, Lund SP, Mangold L, Isharwal S, Christudass CS, Partin AW, Humphreys EB, Sorbara L, Srivastava S, Wagner PD (2015) Evaluation of two mitochondrial DNA biomarkers for prostate cancer detection. Cancer Biomark 15(6):763773. https://doi.org/10.3233/cbm-150518

7. Robinson K, Creed J, Reguly B, Powell C, Wittock R, Klein D, Maggrah A, Klotz L, Parr RL, Dakubo GD (2010) Accurate prediction of repeat prostate biopsy outcomes by a mitochondrial DNA deletion assay. Prostate Cancer Prostatic Dis 13(2):126-131. https://doi.org/10.1038/pcan.2009.64

8. Legisi L, DeSa E, Qureshi MN (2016) Use of the prostate core mitomic test in repeated biopsy decision-making: real-world assessment of clinical utility in a multicenter patient population. Am Health Drug Benefits 9(9):497-502

9. Legros F, Malka F, Frachon P, Lombes A, Rojo M (2004) Organization and dynamics of human mitochondrial DNA. J Cell Sci 117(Pt 13):2653-2662. https://doi.org/10.1242/jcs.01134

10. Chatterjee A, Mambo E, Sidransky D (2006) Mitochondrial DNA mutations in human cancer. Oncogene 25(34):4663-4674. https:// doi.org/10.1038/sj.onc.1209604

11. Johnson PJ, Lo YM (2002) Plasma nucleic acids in the diagnosis and management of malignant disease. Clin Chem 48(8):1186-1193

12. Chatterjee A, Dasgupta S, Sidransky D (2011) Mitochondrial subversion in cancer. Cancer Prev Res (Phila) 4(5):638-654. https:// doi.org/10.1158/1940-6207.capr-10-0326

13. Gnanapragasam VJ, Burling K, George A, Stearn S, Warren A, Barrett T, Koo B, Gallagher FA, Doble A, Kastner C, Parker RA (2016) The prostate health index adds predictive value to multi-parametric MRI in detecting significant prostate cancers in a repeat biopsy population. Sci Rep 6:35364. https://doi. org/10.1038/srep35364

14. Kuru TH, Roethke MC, Rieker P, Roth W, Fenchel M, Hohenfellner M, Schlemmer HP, Hadaschik BA (2013) Histology corespecific evaluation of the European Society of Urogenital Radiology (ESUR) standardised scoring system of multiparametric magnetic resonance imaging (mpMRI) of the prostate. BJU Int 112(8):1080-1087. https://doi.org/10.1111/bju.12259

15. Epstein JI, Allsbrook WC Jr, Amin MB, Egevad LL, Committee IG (2005) The 2005 International Society of Urological Pathology (ISUP) Consensus Conference on Gleason Grading of Prostatic Carcinoma. Am J Surg Pathol 29(9):1228-1242

16. Andriole GL, Levin DL, Crawford ED, Gelmann EP, Pinsky PF, Chia D, Kramer BS, Reding D, Church TR, Grubb RL, Izmirlian G, Ragard LR, Clapp JD, Prorok PC, Gohagan JK, Team PP (2005) Prostate cancer screening in the prostate, lung, colorectal and ovarian (PLCO) cancer screening trial: findings from the initial screening round of a randomized trial. J Natl Cancer Inst 97(6):433-438. https://doi.org/10.1093/jnci/dji065

17. Reznik E, Miller ML, Senbabaoglu Y, Riaz N, Sarungbam J, Tickoo SK, Al-Ahmadie HA, Lee W, Seshan VE, Hakimi AA, Sander C (2016) Mitochondrial DNA copy number variation across human cancers. Elife. https://doi.org/10.7554/elife.10769

18. Mengel-From J, Thinggaard M, Dalgard C, Kyvik KO, Christensen K, Christiansen L (2014) Mitochondrial DNA copy number in peripheral blood cells declines with age and is associated with general health among elderly. Hum Genet 133(9):1149-1159. https://doi.org/10.1007/s00439-014-1458-9

19. Welch HG, Fisher ES, Gottlieb DJ, Barry MJ (2007) Detection of prostate cancer via biopsy in the Medicare-SEER population during the PSA era. J Natl Cancer Inst 99(18):1395-1400. https:// doi.org/10.1093/jnci/djm119 
20. Crawford D, Shore N, Cooperberg M, Dall'Era M, G La Rosa F (2017) Clinical considerations after a negative prostate biopsy. J Prostate Cancer 2:114

21. Lu AJ, Syed JS, Nguyen KA, Nawaf CB, Rosoff J, Spektor M, Levi A, Humphrey PA, Weinreb JC, Schulam PG, Sprenkle PC (2017) Negative multiparametric magnetic resonance imaging of the prostate predicts absence of clinically significant prostate cancer on 12-core template prostate biopsy. Urology 105:118-122. https://doi.org/10.1016/j.urology.2017.01.048

22. Ong WL, Weerakoon M, Huang S, Paul E, Lawrentschuk N, Frydenberg M, Moon D, Murphy D, Grummet J (2015) Transperineal biopsy prostate cancer detection in first biopsy and repeat biopsy after negative transrectal ultrasound-guided biopsy: the victorian transperineal biopsy collaboration experience. BJU Int 116(4):568-576. https://doi.org/10.1111/bju.13031

23. Lopez-Corona E, Ohori M, Wheeler TM, Reuter VE, Scardino PT, Kattan MW, Eastham JA (2006) Prostate cancer diagnosed after repeat biopsies have a favorable pathological outcome but similar recurrence rate. J Urol 175(3 Pt 1):923-927. https://doi. org/10.1016/s0022-5347(05)00350-2 (discussion 927-928)

24. Glinge C, Clauss S, Boddum K, Jabbari R, Jabbari J, Risgaard B, Tomsits P, Hildebrand B, Kaab S, Wakili R, Jespersen T, TfeltHansen J (2017) Stability of circulating blood-based microRNAs-pre-analytic methodological considerations. PLoS One 12(2):e0167969. https://doi.org/10.1371/journal.pone.0167969

25. Loeb S, Sokoll LJ, Broyles DL, Bangma CH, van Schaik RH, Klee GG, Wei JT, Sanda MG, Partin AW, Slawin KM, Marks LS, Mizrahi IA, Shin SS, Cruz AB, Chan DW, Roberts WL, Catalona WJ (2013) Prospective multicenter evaluation of the Beckman
Coulter Prostate Health Index using WHO calibration. J Urol 189(5):1702-1706. https://doi.org/10.1016/j.juro.2012.11.149

26. Parekh DJ, Punnen S, Sjoberg DD, Asroff SW, Bailen JL, Cochran JS, Concepcion R, David RD, Deck KB, Dumbadze I, Gambla M, Grable MS, Henderson RJ, Karsh L, Krisch EB, Langford TD, Lin DW, McGee SM, Munoz JJ, Pieczonka CM, Rieger-Christ K, Saltzstein DR, Scott JW, Shore ND, Sieber PR, Waldmann TM, Wolk FN, Zappala SM (2015) A multi-institutional prospective trial in the USA confirms that the $4 \mathrm{~K}$ score accurately identifies men with high-grade prostate cancer. Eur Urol 68(3):464-470. https://doi.org/10.1016/j.eururo.2014.10.021

27. Lazzeri M, Haese A, de la Taille A, Palou Redorta J, McNicholas T, Lughezzani G, Scattoni V, Bini V, Freschi M, Sussman A, Ghaleh B, Le Corvoisier P, Alberola Bou J, Esquena Fernandez S, Graefen M, Guazzoni G (2013) Serum isoform [-2]proPSA derivatives significantly improve prediction of prostate cancer at initial biopsy in a total PSA range of 2-10 ng/ml: a multicentric European study. Eur Urol 63(6):986-994. https://doi.org/10.1016/j. eururo.2013.01.011

28. Wang FB, Chen R, Ren SC, Shi XL, Zhu YS, Zhang W, Jing TL, Zhang C, Gao X, Hou JG, Xu CL, Sun YH (2017) Prostate cancer antigen 3 moderately improves diagnostic accuracy in Chinese patients undergoing first prostate biopsy. Asian J Androl 19(2):238-243. https://doi.org/10.4103/1008-682x.167715

29. Thompson IM, Ankerst DP, Chi C, Lucia, Goodman PJ, Crowley JJ, Parnes HL, Coltman CA Jr (2005) Operating characteristics of prostate-specific antigen in men with an initial PSA level of $3.0 \mathrm{ng} / \mathrm{ml}$ or lower. JAMA 1(1):66-70. https://doi.org/10.1001/ jama.294.1.66 